\title{
ENHANCED LOCATION BASED SELF-ADAPTIVE ROUTING ALGORITHM FOR WSN IN INDUSTRIAL AUTOMATION
}

\author{
Supriya S. Kamble ${ }^{1}$, P.C.Bhaskar ${ }^{2}$ \\ ${ }^{1}$ Mtech Student, Electronics Engineering, Department of Technology, Shivaji University, Maharashtra, India \\ ${ }^{2}$ Assist. Professor, Electronics Engineering, Department of Technology Shivaji University, Maharashtra, India
}

\begin{abstract}
Today's most sensitive research area is industrial automation. Traditionally, wired communication used in industrial automation. Nowadays wireless communication increases robustness of network in industrial automation rather than wired. For this purpose use of WSN is done in industrial automation using ZigBee network, AODV routing protocol is supported by network layer of ZigBee network. In this paper a new routing algorithm is proposed which increases packet delivery ratio and reduces routing overhead by full use of location information of nodes and self-adaptation of request zone.
\end{abstract}

Keywords: Wireless sensor network (WSN), Ad-hoc On-Demand Distance Vector (AODV) routing protocol, Route Request (RREQ), Request Zone (Rzone), ZigBee Network, MATLAB, NS-2, PIC18F4550.

\section{INTRODUCTION}

In processing industries, manufacturing industries or chemical industries where human intervention is rare, there is need of automation. If considered chemical industry, it is impossible for human being to go in the plant and take the readings, we need a device that takes readings of that plant and sends data to remote location for controlling purpose. In this situation industrial automation is needed and for this reason WSN is created in the industry.

The WSN is composed of more than one small sensor. These small sensors are consists of sensing, data processing and communication capabilities [1].

For creation of WSN most popular standard used is ZigBee network and Ad-Hoc On-demand Distance Vector (AODV) routing protocol [2,3] is supported by ZigBee network layer which is event-driven [4]. When node has data packets to send then only it searches a route from the source to the destination otherwise the nodes are remain in sleep mode. The AODV uses flooding as default routing technique for route discovery [2,3]; due to flooding packets in the network, contention and collision occur in the

MAC layer as MAC 802.15.4 cannot performs heavy routing overhead causes a broadcast storm as a result packet delivery ratio reduced [4].

To increase packet delivery ratio and reduce routing overhead, in this paper proposed a new routing algorithm which is 'Enhanced Location Based Self- Adaptive Routing Algorithm for WSN'. For route discovery full use of location information is done rather searching route blindly using flooding.
The main features of IEEE 802.15.4's MAC and PHY layers are presented in[5]; PHY layer tradeoffs, primitives, packet structure and topology, super frame of MAC layer these special features of IEEE 802.15.4 with the ZigBee Network layer routing.

The performance evaluations of IEEE 802.15.4 of the new medium access protocol was done by simulation which is star topology beacon-enabled mode with super frame structure features used to allow devices for accessing channels in a contention access period (CAP) or a collision free period (CFP) and the beacon-based synchronization mechanism. Also provided an analysis comparison of the energy costs of beacon tracking and non- tracking modes for synchronization that shows the optimum choice depends upon the combination of duty cycles and data rates[6].

In paper [7] presented a survey of different routing techniques with design challenges for routing protocols in WSNs. There are three routing techniques as flat, hierarchical and location-based routing. Depends on protocol operation these protocols can be classified into multipath, query, negotiation, QoS and coherent based. And the design tradeoffs between energy and communication overhead savings in every routing model with performance issues and advantages of every routing technique.

The schemes for scalable routing protocols are loop-free, localized and follow a single-path strategy. Routing protocols having two modes: greedy mode which is used if and only if source node is able to forward the message to the destination and recovery mode applied greedy mode is available. By use of different metrics such as hop count, power, cost, congestion etc and in past traffic memorization at nodes that are memory less or memorizing past traffic makes difference in both methods [8]. 
In the Linux operating system an implementation done using simulation. Certain modifications done in AODV and the Linux kernel for converting the simulation into an implementation for making simplification in the simulation of AODV and the Linux kernel incompatibilities and in a mobile environment routing for the IP-layer[9].

\section{PROPOSED ALGORITHM}

The proposed algorithm uses full location information of node when any node wants to transmit data. At that time by broadcasting route request packet (RREQ) in the request zone route discovery initiated by source node $S$ as existing route is not available when $S$ has data to send to destination node D.

When route request packet reached to $\mathrm{D}$ or any intermediate at that instant route reply (RREP) is given on reverse link.

The proposed algorithm uses location information for route discovery of AODV by creating request zone and routing overhead reduced due to limited search area to request zone.

\section{ROUTING ALGORITHM}

\subsection{Ad Hoc on -Demand Distance Vector (AODV)}

\section{Routing Protocol}

The different routing protocols used for wireless communication, AODV are one of them. For establishing and maintaining an ad hoc network the AODV activates dynamic, self-starting, multihop routing between participating mobile nodes. Using the lost link AODV able to invalidate the routes by notified the affected set of nodes when links break. It is having four different types of packets[15] such as Route Request, Route Reply, Hello and Route Error.

AODV can work with unicast, broadcast, multicast communication in ad hoc mobile networks. A source node is needed a route then only route discovery initiated by AODV. By the use of sequence numbers routes are maintained long time. The next hop routing information for destination node is maintained in route table of AODV node[7].

\subsection{Location Based Route Discovery:}

The addresses of the source node and the destination node are stored in the route request packet when the Rzone is defined. The recvRREQ algorithm of location based selfadaptive routing algorithm for WSN will be executed to forward the RREQ as each intermediate node $X 0$ receives an RREQ as Algorithm 1 shows. In recvRREQ algorithm, the static nodes inside the Rzone are responsible for rebroadcasting an RREQ. The RREQ is directly discarded by the mobile nodes if it is not the destination and it acts as intermediate node.

As the definition of the Rzone directly influences the performance of enhanced location based self-adaptive routing algorithm for WSN; the proper Rzone can reduce the number of broadcast RREQs and save bandwidth and energy.

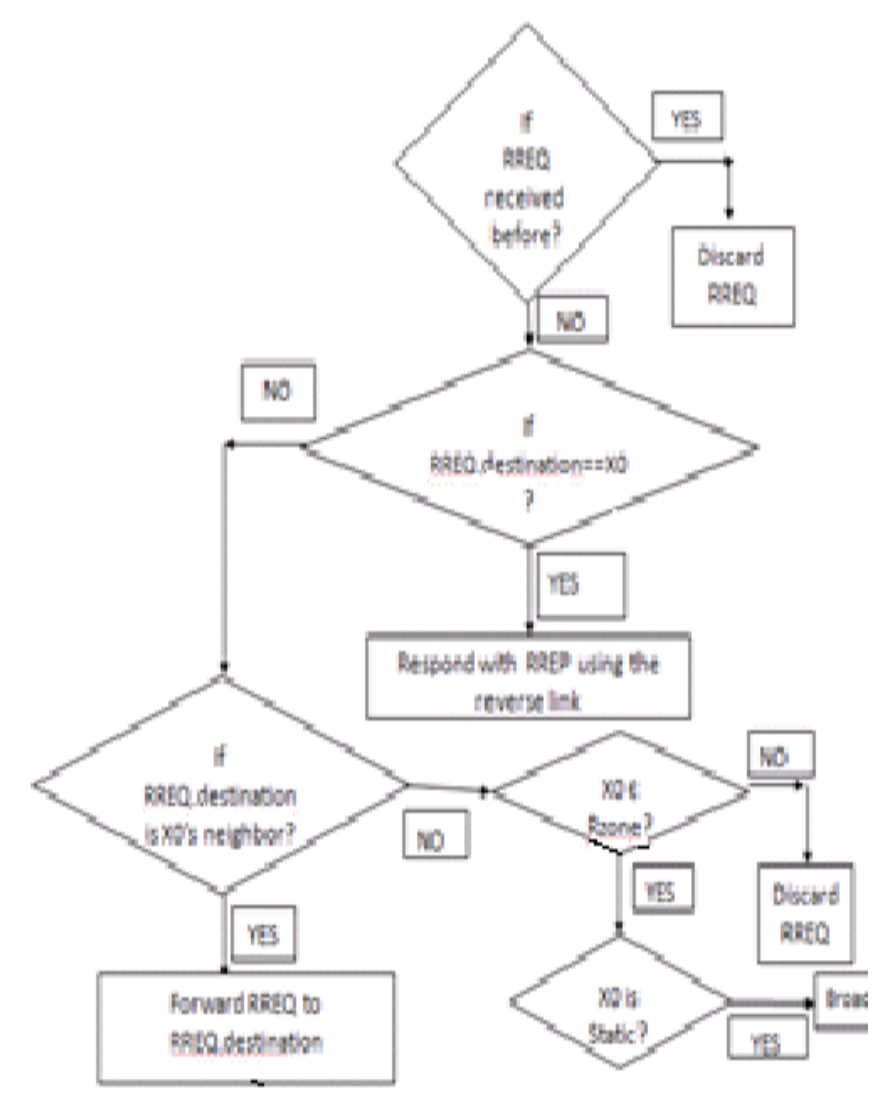

Algorithm 1 recvRREQ algorithm[5]

\section{HARDWARE IMPLEMENTATION}

\subsection{Hardware at Each Node}

As shown in figure 1, hardware at each node includes sensor, PIC18F4550, laptop and ZigBee Module.

\section{SOFTWARE IMPLEMENTATION}

\subsection{Software for Initialization of Hardware}

Sensor data should be digitized, for this purpose sensor data given to PIC18F4550 then by UART provided for executing algorithm to laptop at the end transmission given to the ZigBee module. Hence, once the sensor data is get this is to be first digitized for this data taken by ADC of PIC18f4550. This digitized signal is given to UART of PIC also displayed on LCD. This initialization of ADC, UART and LCD is done using MPLAB8.0.

Then data at UART is transmitted to laptop to execute the algorithm in MATLAB2013a at the end sent to ZigBee module to transmit an appropriate node. ZigBee module is used in broadcast mode and initialized using AT commands. 
Once data received by appropriate node, it sends acknowledgment to the source node.

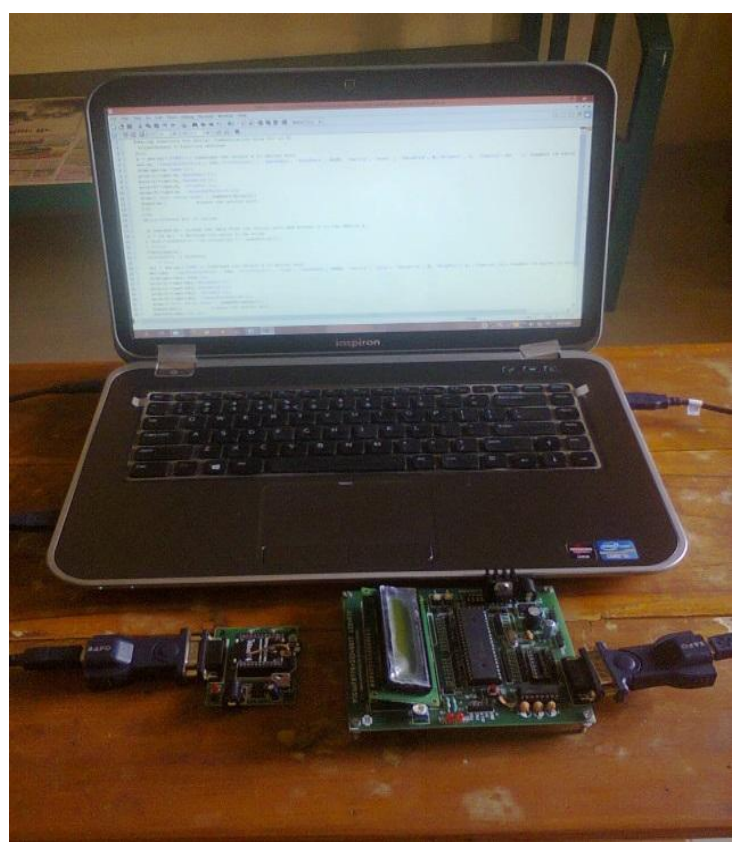

Fig 1 A sensor node

\subsection{Enhancement in LBAR}

In self-adaptive routing considers the node location for zone. At the same time link reliability in terms of energy consumption and residual energy can be considered.

In a particular communication all node in the networks transmits their residual energy and according to this residual energy route establishment done. It means that a node having a residual energy more than transmitting energy at that time that node is considered in request zone for route establish otherwise alternative node considered by source node. Due to this the energy consumption is used for route establishment saved.

\section{PERFORMANCE EVALUTION}

Using the NS2 simulation tool a model is developed which simulates performance characteristics of enhanced location based self-adaptive routing algorithm for WSN.

\subsection{Performance Measurement}

The different performance parameters are measured during simulation such as the number of packets sent from source, the number of packets delivered to destination, the number of packets dropped during transmission and energy of each node during transmission.

The source node sends data to destination only when destination belongs to request zone the figure 2 shows the number of packets sent during transmission, figure 3 shows the number of packets delivered during transmission and figure 4 shows the number of packets dropped during transmission. Then figure 5 shows the energy of each node during transmission.

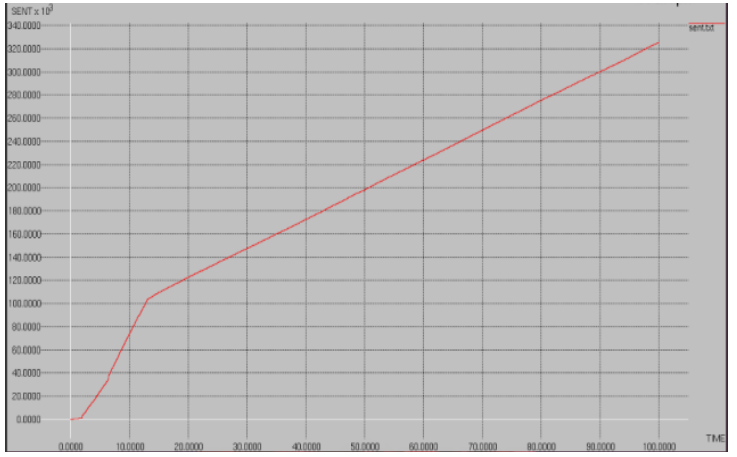

Fig 2 Number of Packets Sent during transmission

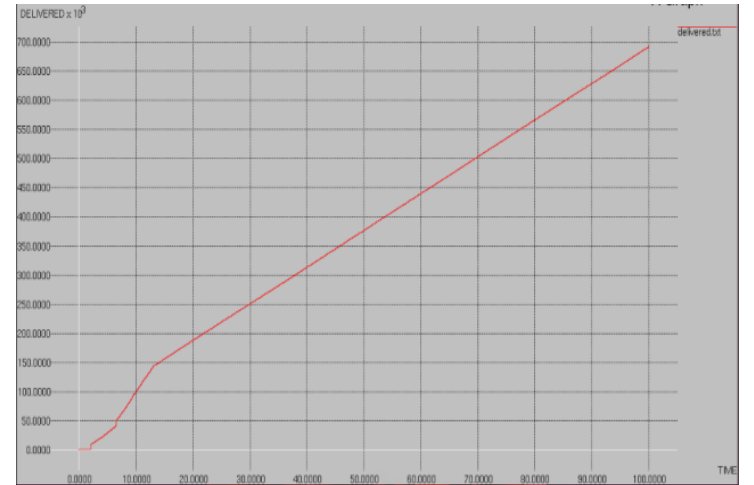

Fig 3 Number of Packets Delivered during transmission

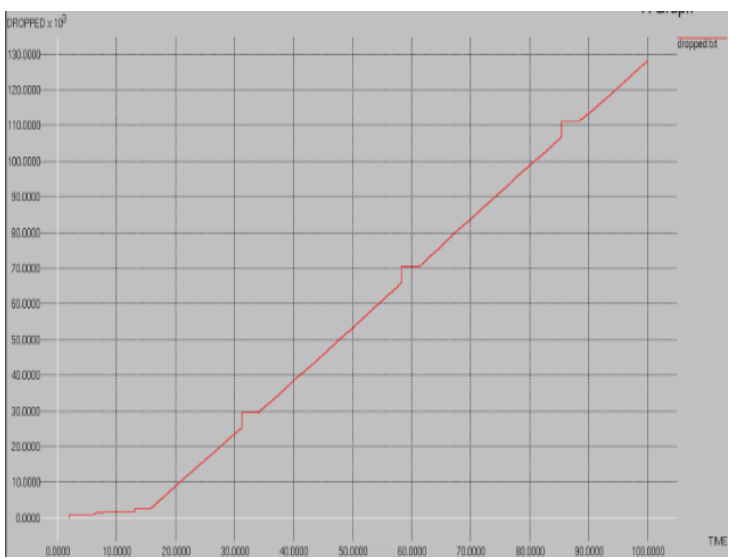

Fig 4 Number of Packets Dropped during transmission

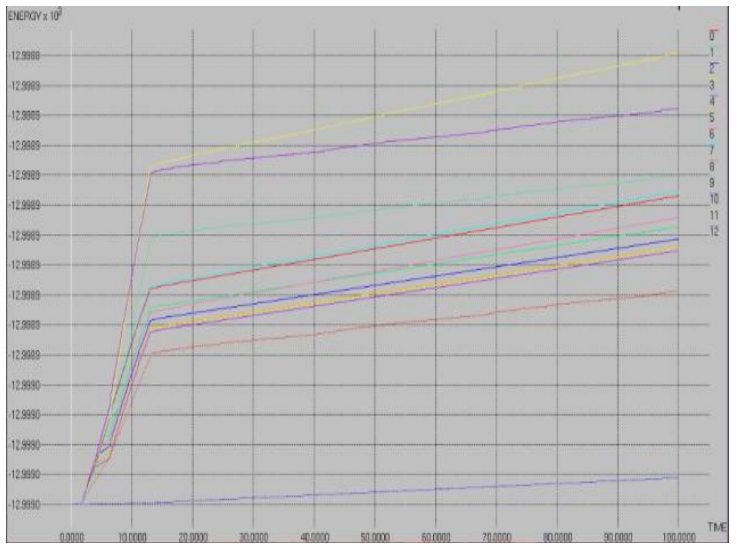

Fig 5: Energy of each node during transmission 


\subsection{Simulation Parameters}

The final simulation results can be influenced by many factors apart from routing algorithm such as number of mobile nodes, number of static nodes.

To design the simulation according to enhanced location based self-adaptive routing algorithm for WSN used simulation parameters shown in Table 1 as below.

Also for realization hardware implementation done by creating WSN using different sensor nodes such as temperature, humidity and light intensity sensor and ZigBee module is used as transmission media.

Figure shows sensor data of different sensors and figure shows data received on ZigBee network shown on XCT-U terminal.

Table 1: Parameters used in simulation

\begin{tabular}{|l|l|}
\hline Parameter & Value \\
\hline MAC protocol & IEEE 802.15.4 \\
\hline Radio propagation model & Two-ray ground model \\
\hline Network Interface type & $\begin{array}{l}\text { Physical } \\
\text { wireless/802.15.4 }\end{array}$ \\
\hline Interface queue & Priority queue \\
\hline Queue length & 150 \\
\hline Routing protocol & AODV \\
\hline $\begin{array}{l}\text { X and Yimensions of } \\
\text { topography }\end{array}$ & $500 * 500$ sqm \\
\hline Initial energy of the node & 13000 Joules \\
\hline $\begin{array}{l}\text { Transmitting power of the } \\
\text { Node }\end{array}$ & 0.0744 Watts \\
\hline $\begin{array}{l}\text { Receiving power of the } \\
\text { Node }\end{array}$ & 0.0648 Watts \\
\hline Idle power of the node & 0.00000552 Watts \\
\hline Traffic type & Constant Bit Rate (CBR) \\
\hline Packet size & 70 Bytes \\
\hline Data Interval & 0.045 second \\
\hline
\end{tabular}

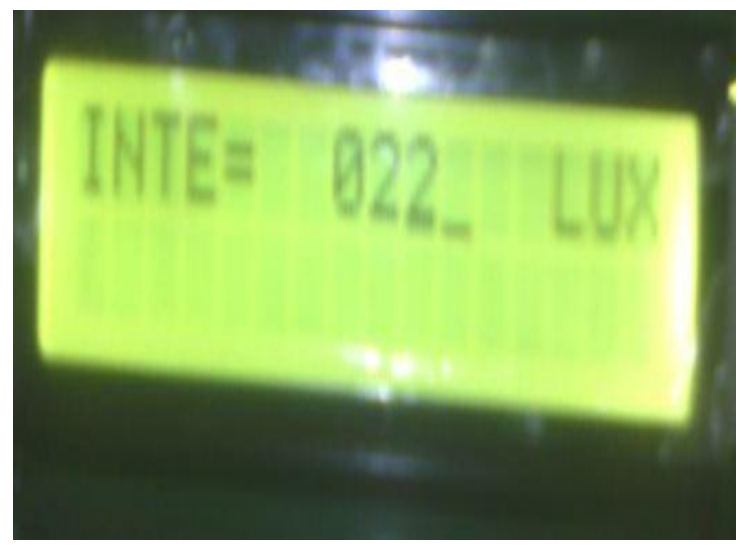

Fig 6: Sensor digitized data displayed on LCD

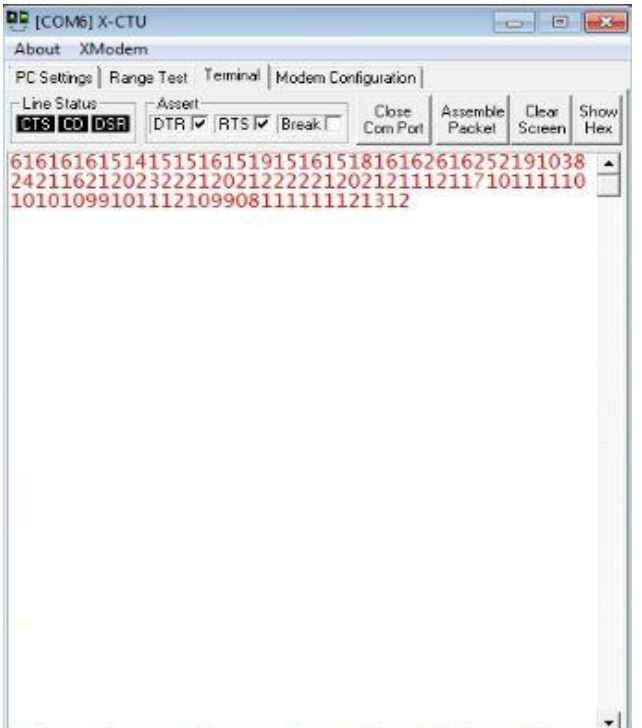

Fig 7: After execution of recvRREQ algorithm transmitted data to destination node.

\section{CONCLUSIONS}

In this paper developed new algorithm which is based on AODV, ZigBee network and WSN industrial automation is location based self-adaptive routing algorithm. It uses full location information for route discovery by broadcasting route request in the request zone due to this routing overhead reduced.

Also enhancement done in location based self-adaptive routing algorithm in terms of energy consumption and the residual energy.

\section{REFERENCES}

[1] I.F.Akyildiz, W.Su,Y.Sankara Subramanian, and E. Cayirci, "Wireless sensor networks: a survey," Computer Networks, vol. 38, no. 4, pp. 393-422, 2002.

[2] C. Perkins, Nokia Research Center, E. Belding-Royer University of California, Santa Barbara, S. Das University of Cincinnati, "Ad hoc On-Demand Distance Vector (AODV) Routing”, July 2003.

[3] "Ad hoc On Demand Distance Vector (AODV) Routing Protocol", Dr. Baruch Awerbuch\& Dr. Amitabh Mishra.

[4] Xiao Hui Li, SeungHo Hong, and Kang Ling Fang, "Location-Based Self-Adaptive Routing Algorithm for Wireless Sensor Networks in Home Automation".

[5] ZigBee Specification, ZigBee Alliance Std. Document 053474r17, 2007.

[6] Gang Lu, Bhaskar Krishnama Chari, Cauligi S. Ragha vendra "Performance Evaluation of the IEEE 802.15.4 MAC for Low-Rate Low-Power Wireless Networks".

[7] J. N. Al-Karaki and A. E. Kamal, "Routing techniques in wireless sensor networks: a survey," IEEE Wireless Communications, vol. 11, no. 6, pp. 6-27, 2004. 
[8] Stojmenovic, "Position-based routing in ad hoc networks," IEEE Communications Magazine, vol. 40, no. 7, pp. 128-134, 2002.

[9] Elizabeth M. Royer and Charles E. Perkins ,"An Implementation Study of the AODV Routing Protocol".

[10] "Introduction to Network Simulator NS2", Teerawat Issariyakul and Ekram Hossain

[11] I. D. Chakeres and K. B. Luke, "AODVjr, AODV simplified," Mobile Computing and Communications Review, vol. 6, no. 3, pp. 100-101, 2002.

[12] K. Akkaya and M. Younis, "A survey on routing protocols for wireless sensor networks," Ad Hoc Networks, vol. 3, no. 3, pp. 325-349, 2005.

[13] J. L. Zheng and M. J. Lee, "Sensor Network Operations: A Comprehensive Performance Study of IEEE 802.15.4”, McGraw-Hill, New York, NY, USA, 2006.

[14] C. Chen and J. Ma, "Simulation study of AODV performance over IEEE 802.15.4 MAC in WSN with mobile sinks," in Proceedings of the $21 \mathrm{st}$ International Conference on AdvancedInformation Networking and Applications Workshops (AINAW'07), vol. 1, pp. 159-164, Niagara Falls, Canada, May 2007.

[15] Jason Hill, Mike Horton, Ralph Kling and Lakshman Krishnamurthy, "The Platforms Enabling Wireless Sensor Networks".

[16] T. Sun, L.-J. Chen, C.-C. Han, G. Yang, and M. Gerla, "Measuring effective capacity of IEEE 802.15.4 beaconless mode," in Proceedings of IEEE Wireless Communications and Networking Conference (WCNC '06), vol. 1, pp. 493-498, Las Vegas, Nev, USA, April 2006.

[17] "Location-based Routing in Sensor Networks II", JieGao

[18] "Position Based Routing for Wireless Mobile Ad Hoc Networks", Geetam S. Tamar

[19] "Adaptive Routing Algorithms and Implementations", David Ouellet-Poulin

[20] DelphineChristin, Parag S. Mogre and Matthias Hollick, "Survey on Wireless Sensor Network Technologies for Industrial Automation: The Security and Quality of Service Perspectives".

[21] Shripad V Deshpande\& P. R. Devale, "Recent Trends in using Wireless Sensor Networks in Industrial Environment".

[22] Gang Zhao, "Wireless Sensor Networks for Industrial Process Monitoring and Control: A Survey",Network Protocols and Algorithms, ISSN 1943-3581,2011, Vol. 3, No. 1

[23] L. Q. Zhuang, K. M. Goh and J. B. Zhang, "The Wireless Sensor Networks for Factory Automation: Issues and Challenges"

[24] "Routing Protocols in Wireless Sensor Networks",Luis Javier García Villalba, Ana Lucila Sandoval Orozco, Alicia Triviño Cabrera and CláudiaJacyBarenco Abbas 